\title{
Chiral extrapolations and strangeness in the baryon ground states
}

\author{
Matthias F.M. Lutz \\ GSI Helmholtzzentrum für Schwerionenforschung GmbH \\ Planckstr. 1, 64291 Darmstadt, Germany \\ E-mail: m. lutzegsi.de \\ Alexander Semke \\ GSI Helmholtzzentrum für Schwerionenforschung $\mathrm{GmbH}$ \\ Planckstr. 1, 64291 Darmstadt, Germany \\ E-mail: a.semke@gsi.de
}

\begin{abstract}
We review the quark-mass dependence of the baryon octet and decuplet masses as obtained from recent lattice simulations of the BMW, PACS-CS, LHPC, HSC and QCDSF-UKQCD groups. Our discussion relies on the relativistic chiral Lagrangian and large- $N_{c}$ sum rule estimates of the counter terms relevant for the baryon masses at $\mathrm{N}^{3} \mathrm{LO}$. A partial summation is implied by the use of physical baryon and meson masses in the one-loop contributions to the baryon self energies. In our analysis the physical masses are reproduced exactly by means of a suitable set of linear constraints. A quantitative and simultaneous description of all lattice results is achieved in terms of a six parameter fit, where the symmetry conserving counter term that are relevant at $\mathrm{N}^{3} \mathrm{LO}$ are not yet being used. For pion masses larger than $300 \mathrm{MeV}$ there appears to be an approximate linear pion-mass dependence of all octet and decuplet baryon masses. We discuss the pion- and strangeness sigma terms of the baryon octet states.
\end{abstract}

The 7th International Workshop on Chiral Dynamics,

August 6-10, 2012

Jefferson Lab, Newport News, Virginia, USA

${ }^{*}$ Speaker. 


\section{Introduction}

The purpose of this talk is to review the chiral extrapolation and strangeness content of the baryon octet and decuplet ground states. We discuss the recent lattice data on the pion-mass dependence of the baryon masses [1, 2, 3, 4, 5, 6, 7, 8]. Given a systematic analysis based on the chiral Lagrangian, the strange-quark mass dependence of the baryon masses can be calculated. Such results scrutinize the consistency of the chiral extrapolation approach and lattice simulations of the baryon masses. Variations of the baryon masses along suitable pathes in the pion-kaon mass plane are important to improve the determination of the low-energy parameters of QCD as encoded into the chiral Lagrangian.

Our work relies on the relativistic chiral Lagrangian with baryon octet and decuplet fields where effects at $\mathrm{N}^{3} \mathrm{LO}$ (next-to-next-to-next-leading order) are considered systematically. The details of the approach are published in $[9,10,11,12,13]$. The chiral extrapolation of baryon masses with strangeness content is critically discussed in the literature $[14,15,10,2,16,17,18,19$, $11,7]$. The convergence properties of a strict chiral expansion for the baryon masses with three light flavors are very poor, if existing at all for a physical strange quark mass. Different strategies how to perform partial summations or phenomenological adaptations are investigated by several groups $[9,10,15,17,16]$. A straight forward application of chiral perturbation theory to recent QCD lattice simulations appears futile (see e.g. [1, 2, 16, 7]). A crucial element of our scheme is the use of physical baryon masses in the one-loop contribution to the baryon self energies. Furthermore, the low-energy constants required at $\mathrm{N}^{3} \mathrm{LO}$ are estimated by sum rules that follow from QCD in the limit of a large number of colors $\left(N_{c}\right)$ [20,11]. Since we obtain a simultaneous and quantitative description of the lattice data of the BMW, PACS-CS, LHPC, HSC and QCDSF-UKQCD groups, we find it justified to present detailed results on the strange-quark mass dependence of all members of the baryon octet and decuplet states. In particular, we confront our parameter set against recent analyses of the BMW and QCDSF-UKQCD groups on the pion- and strangeness-sigma terms of the baryon octet states $[6,22,23]$.

\section{Chiral extrapolation of baryon masses}

We consider the chiral extrapolation of the baryon masses to unphysical quark masses. Assuming exact isospin symmetry, the hadron masses are functions of $m_{u}=m_{d} \equiv m_{q}$ and $m_{s}$. The dependence on the light quark masses may be traded against a dependence on the pion and kaon masses. The 'physical' strange quark mass is determined such that at the physical pion mass the empirical kaon mass is reproduced. Our approach is detailed in $[9,10,11]$. In particular, we assume a quark-mass dependence of the pion and kaon masses as predicted by $\chi \mathrm{PT}$ at the next-to-leading order with parameters as recalled in [11]. The baryon masses are computed at $\mathrm{N}^{3} \mathrm{LO}$ where we use physical baryon and meson masses in the one-loop contributions to the baryon self energies and assume systematically large- $N_{c}$ sum rules for the parameter set.

Initially we adjusted the parameter set to the physical masses of the baryon octet and decuplet states and to the results for the pion-mass dependence of the nucleon and omega masses as predicted by the BMW group [4]. Using the isospin average of the empirical baryon masses we derived a system of linear equations which was used to express eight low-energy constants in terms of the 

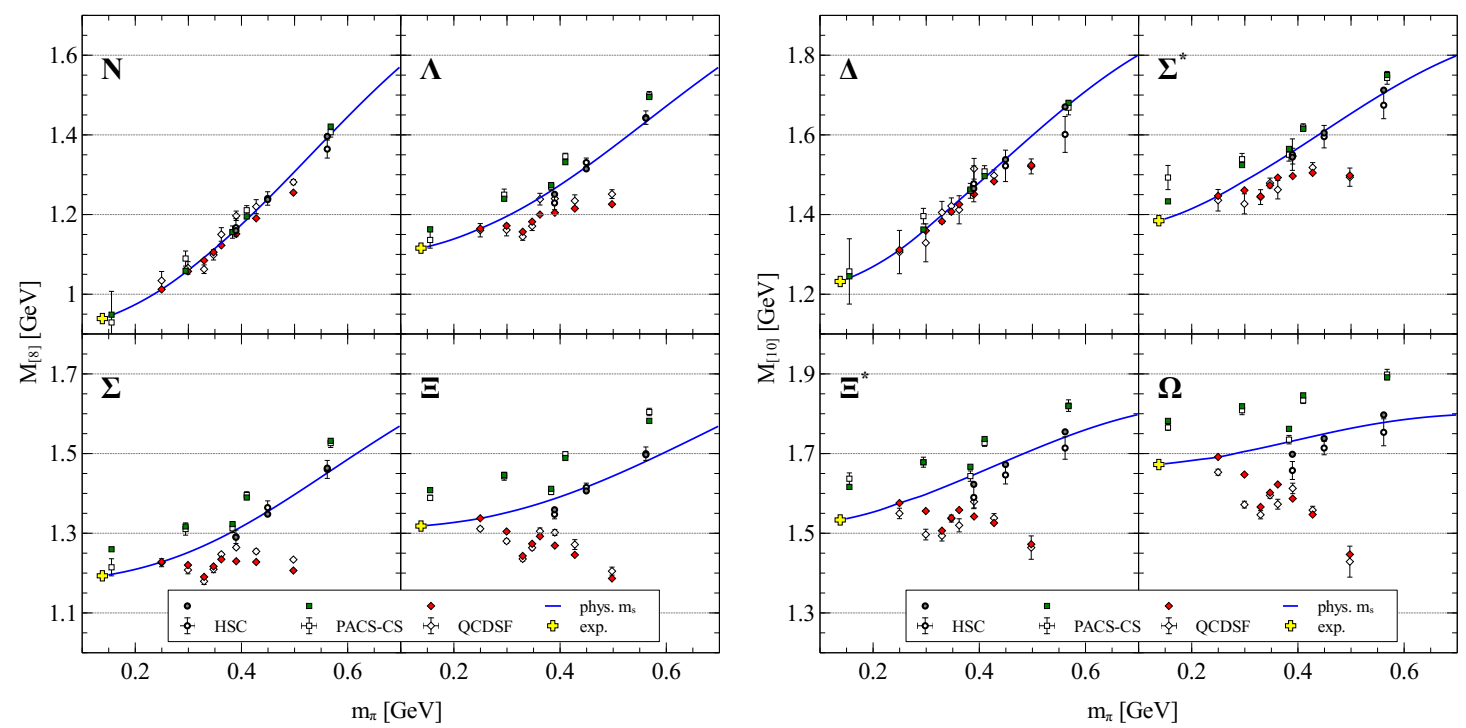

Figure 1: Baryon masses as a function of the pion mass as explained in the text.

remaining parameters. This provided a significant simplification of the parameter determination and allowed us to analyze the set of non-linear and coupled equations in great depth [11]. An accurate reproduction of the physical baryon masses and lattice data was achieved. All parameters, except of the symmetry preserving counter terms relevant at $\mathrm{N}^{3} \mathrm{LO}$, were considered. Given the large- $N_{c}$ sum rules of [20], there are 5 independent parameters only, which are all set to zero so far. The latter have a rather minor effect on the baryon masses and can be determined only with very precise lattice data. That initial parameter set was further slightly adjusted in [12] to achieve consistency with recent lattice results of the LHPC and PACS-CS groups [7, 2]. Insisting on a simultaneous description of the BMW and PACS-CS data we unambiguously recover the unfitted results of the HSC and QCDSF-UKQCD groups [3, 8] with an amazing accuracy [13]. We also explored the role of the axial coupling constants $F$ and $D$, which we found to be accurately determined from a simultaneous fit of the lattice data [13].

In Fig. 1 we show the baryon masses for our favored parameter set 3 as a function of the linear pion mass [12]. It is a striking phenomena that for pion masses larger than $300 \mathrm{MeV}$ there appears to be an approximate linear dependence for all baryon masses. Note however, that given our approach the linear dependence is significantly and systematically altered at smaller pion masses. Such a behavior was discussed in the talk of Walker-Loud at this conference based on various lattice data for the nucleon mass. Our results are confronted against the lattice data from the HSC groups (open circles), which provide data for an almost physical strange quark mass. In order to provide a quantitative comparison, we compute the baryon masses for the pion and kaon masses as predicted by the HSC group. The grey filled circles show our results. The distance of the filled circles to the solid lines measures the importance of taking the precise physical strange quark mass in the computation of the baryon masses. In Fig. 1 we also confront our approach with the simulation data of the PACS-CS (open squares) and QCDSF-UKQCD (open diamonds) groups. Since the later groups use strange quark masses that are significantly off the physical value, the 
data points are typically quite distant from the solid line. Again our results, green squares and red diamonds, are quite close to their corresponding lattice points, open squares and diamonds. Note that all lattice points are shown with statistical errors only, where we assume the central values of the lattice spacing as provides by the various lattice groups.

\section{Pion and strangeness baryon sigma terms}

The pion- and strangeness baryon sigma terms play an important role in various physical systems. For instance, the pion-nucleon sigma term is of greatest relevance in the determination of the density dependence of the quark condensate at low baryon densities and therefore provides a first estimate of the critical baryon density at which chiral symmetry may be restored (see e.g. [24]). Similarly, the kaon-nucleon sigma terms are key parameters for the determination of a possible kaon condensate in dense nuclear matter [25].

Assuming exact isospin symmetry with $m_{u}=m_{d} \equiv m_{q}$, the pion-nucleon sigma term reads

$$
\sigma_{\pi N}=m_{q}\langle N(p)|\bar{q} q| N(p)\rangle=m_{q} \frac{\partial}{\partial m_{q}} m_{N} .
$$

The pion-sigma terms of the remaining baryon states are defined analogously to (3.1). As indicated in (3.1), the matrix elements of the scalar quark operator are accessible via the derivative of the nucleon mass with respect to the light quark mass $m_{q}$. This follows directly from the FeynmanHellman theorem. The strangeness sigma term $\sigma_{s N}$ of the nucleon is determined by the derivative of the nucleon mass with respect to the strange quark mass $m_{s}$ :

$$
\sigma_{s N}=m_{s}\langle N(p)|\bar{s} s| N(p)\rangle=m_{s} \frac{\partial}{\partial m_{s}} m_{N} .
$$

In Tab. 1 we present our predictions for the pion- and strangeness sigma terms of the baryon octet states for various parameter set as explained in [12]. They are compared with two recent lattice determinations $[6,22]$. Our values for the non-strange sigma terms are in reasonable agreement with the lattice results. In particular, we obtain a rather small value for the pion-nucleon sigma term, which is within reach of the seminal result $\sigma_{\pi N}=45 \pm 8 \mathrm{MeV}$ of Gasser, Leutwyler and Sainio in

\begin{tabular}{c||l|c|c|cccc}
\hline & {$[6]$} & {$[22]$} & {$[16]$} & Fit 1 & Fit 2 & Fit 3 & Fit 4 \\
\hline \hline$\sigma_{\pi N}$ & $39(4)_{-7}^{+18}$ & $31(3)(4)$ & $59(2)(17)$ & 34 & 33 & 31 & 31 \\
$\sigma_{\pi \Lambda}$ & $29(3)_{-5}^{+11}$ & $24(3)(4)$ & $39(1)(10)$ & 24 & 21 & 20 & 20 \\
$\sigma_{\pi \Sigma}$ & $28(3)_{-3}^{+19}$ & $21(3)(3)$ & $26(2)(5)$ & 17 & 15 & 14 & 14 \\
$\sigma_{\pi \Xi}$ & $16(2)_{-3}^{+8}$ & $16(3)(4)$ & $13(2)(1)$ & 11 & 7 & 7 & 7 \\
\hline \hline$\sigma_{s N}$ & $34(14)_{-24}^{+28}$ & $71(34)(59)$ & $-4(23)(25)$ & 43 & 24 & 2 & 3 \\
$\sigma_{s \Lambda}$ & $90(13)_{-38}^{+24}$ & $247(34)(69)$ & $126(26)(35)$ & 238 & 194 & 191 & 194 \\
$\sigma_{s \Sigma}$ & $122(15)_{-36}^{+25}$ & $336(34)(69)$ & $159(27)(45)$ & 311 & 291 & 273 & 278 \\
$\sigma_{s \Xi}$ & $156(16)_{-38}^{+36}$ & $468(35)(59)$ & $267(31)(50)$ & 449 & 380 & 407 & 408 \\
\hline
\end{tabular}

Table 1: Pion- and strangeness sigma terms of the baryon octet states in units of MeV. 
[26]. The size of the pion-nucleon term can be determined from the pion-nucleon scattering data. It requires a subtle subthreshold extrapolation of the scattering data. Despite the long history of the sigma-term physics, the precise determination is still highly controversial (for one of the first reviews see e.g. [27]). Such a result is also consistent with the recent analysis of the QCDSF collaboration [23], which suggests a value $\sigma_{\pi N}=38 \pm 12 \mathrm{MeV}$. Our estimate for the strangeness sigma term of the nucleon with $\sigma_{s N} \simeq 22 \pm 20 \mathrm{MeV}$ is compatible with the currently most precise lattice prediction $\sigma_{s N}=12_{-16}^{+23} \mathrm{MeV}$ in [23]. For the strangeness sigma terms of the remaining octet states there appears to be a striking conflict amongst the values obtained by the BMW and QCDSF-UKQCD groups. Our values are quite compatible with the former. In Tab. 1 we recall also the results of a chiral extrapolation of the recent PACS-CS data by Camalich et al. [16]. The analysis is based on the baryon masses truncated at $\mathrm{N}^{2} \mathrm{LO}$ with phenomenologically adjusted values for the meson-baryon coupling constants. For almost all sigma terms we find significant differences to our results. This may reflect the significantly much less accurate reproduction of the PACS-CS data and the physical baryon masses in [16].

\section{Summary and outlook}

In this work we reviewed the results of five different lattice groups, BMW, LHPC, PACS-CS, HSC and QCDSF-UKQCD on the baryon octet and decuplet masses. Using the chiral Lagrangian at $\mathrm{N}^{3} \mathrm{LO}$ we obtained a universal parameter set that leads to a quantitative reproduction of the baryon masses and all lattice simulation data taken at quite different pairs of pion and kaon masses. While the physical masses are reproduced exactly, the lattice data are successfully fitted with six free parameters only. The parameters may be adjusted to the pion-mass dependence of the nucleon and omega masses of the BMW group together with the masses of all baryon octet and decuplet states of the PACS-CS group only. In turn, the simulation data of the HSC and QCDSF-UKQCD groups are reproduced quite accurately. This suggests a high level of compatibility of the different lattice data sets. For pion masses larger than $300 \mathrm{MeV}$ there appears to be an approximate linear pion-mass dependence of all octet and decuplet baryon masses.

Based on this result, we predicted the pion and strangeness sigma terms of all baryon ground states. In particular, we obtain a quite small pion-nucleon sigma term of about $32 \pm 2 \mathrm{MeV}$ and also for the strangeness sigma term of the nucleon of about $22 \pm 20 \mathrm{MeV}$. Future even more accurate lattice simulations would help to consolidate our parameter set and may lead to a precise determination of the additional five flavor symmetric counter terms that were not considered yet. The latter play a decisive role in meson-baryon scattering processes at next-to-leading order already.

\section{References}

[1] A. Walker-Loud, et al., Light hadron spectroscopy using domain wall valence quarks on an Asqtad sea, Phys. Rev. D79 (2009) 054502.

[2] S. Aoki, et al., 2+1 Flavor Lattice QCD toward the Physical Point, Phys. Rev. D79 (2009) 034503.

[3] H.-W. Lin, et al., First results from $2+1$ dynamical quark flavors on an anisotropic lattice: light-hadron spectroscopy and setting the strange-quark mass, Phys. Rev. D79 (2009) 034502.

[4] S. Durr, et al., Ab-Initio Determination of Light Hadron Masses, Science 322 (2008) 1224-1227. 
[5] C. Alexandrou, et al., Low-lying baryon spectrum with two dynamical twisted mass fermions, Phys.Rev. D80 (2009) 114503.

[6] S. Durr, et al., Sigma term and strangeness content of octet baryons, Phys. Rev. D85 (2012) 014509.

[7] A. Walker-Loud, Evidence for non-analytic light quark mass dependence in the baryon spectrum, arXiv:1112.2658 [hep-lat].

[8] W. Bietenholz, et al., Flavour blindness and patterns of flavour symmetry breaking in lattice simulations of up, down and strange quarks, Phys.Rev. D84 (2011) 054509.

[9] A. Semke, M. F. M. Lutz, Baryon self energies in the chiral loop expansion, Nucl. Phys. A778 (2006) 153.

[10] A. Semke, M. F. M. Lutz, On the possibility of a discontinuous quark-mass dependence of baryon octet and decuplet masses, Nucl. Phys. A789 (2007) 251.

[11] A. Semke, M. F. M. Lutz, Quark-mass dependence of the baryon ground-state masses, Phys. Rev. D85 (2012) 034001.

[12] A. Semke, M. F. M. Lutz, Strangeness in the baryon ground states, Phys. Lett. B717 (2012) 242.

[13] M. F. M. Lutz, A. Semke, On the consistency of recent QCD lattice data of the baryon ground-state masses, Phys. Rev. D86 (2012) 091502.

[14] E. Jenkins, A. V. Manohar, Baryon chiral perturbation theory using a heavy fermion lagrangian, Phys. Lett. B 255 (1991) 558.

[15] M. Frink, U.-G. Meissner, On the chiral effective meson-baryon Lagrangian at third order, Eur. Phys. J. A29 (2006) 255.

[16] J. Martin Camalich, L. S. Geng, M. J. Vicente Vacas, The lowest-lying baryon masses in covariant SU(3)-flavor chiral perturbation theory, Phys. Rev. D82 (2010) 074504.

[17] R. D. Young, A. W. Thomas, Octet baryon masses and sigma terms from an su(3) chiral extrapolation, Phys. Rev. D 81 (2010) 014503.

[18] L.-s. Geng, X.-1. Ren, J. Martin-Camalich, W. Weise, Finite-volume effects on octet-baryon masses in covariant baryon chiral perturbation theory, Phys. Rev. D84 (2011) 074024.

[19] J. Martin Camalich, L. Geng, M. Vicente Vacas, Analysis of the LQCD results on the lowest-lying baryon masses in chiral perturbation theory, AIP Conf.Proc. 1322 (2010) 440.

[20] M. F. M. Lutz, A. Semke, Large-Nc operator analysis of 2-body meson-baryon counterterms in the chiral Lagrangian, Phys. Rev. D83 (2011) 034008.

[21] E. E. Jenkins, A. V. Manohar, J. W. Negele, A. Walker-Loud, A Lattice Test of 1/N(c) Baryon Mass Relations, Phys.Rev. D81 (2010) 014502.

[22] R. Horsley, et al., Hyperon sigma terms for 2+1 quark flavours, arXiv:1110.4971 [hep-lat].

[23] G. S. Bali, et al., The strange and light quark contributions to the nucleon mass from Lattice QCD, arXiv:1111.1600 [hep-lat].

[24] M. F. M. Lutz, B. Friman, C. Appel, Saturation from nuclear pion dynamics, Phys. Lett. B474 (2000) 7-14.

[25] D. Kaplan, A. Nelson, Strange Goings on in Dense Nucleonic Matter, Phys.Lett. B175 (1986) 57-63.

[26] J. Gasser, H. Leutwyler, M. Sainio, Sigma term update, Phys.Lett. B253 (1991) 252-259.

[27] E. Reya, Chiral symmetry breaking and meson - nucleon sigma commutators: A Review, Rev. Mod. Phys. 46 (1974) 545-580. 